\title{
Sequential Decoding on Syndrome Trellises for Nonparametric Detection
}

\author{
Dmitry Osipov ${ }^{1,2}$, Dmitry Titov ${ }^{2}$ \\ ${ }^{1}$ Institute for Information Transmission Problems RAS, 19 bld. 1 Bolshoy Karetny lane, Moscow, \\ Russian Federation \\ ${ }^{2}$ National Research University Higher School of Economics, 3 Kochnovsky Proezd, Moscow, Russian \\ Federation \\ E-mails:d_osipov@iitp.ru titovdim93@gmail.com
}

Abstract: The following paper adapts the classical Zigangirov-Jelinek algorithm to the decoding of nonbinary block codes under severe mixed jamming. To ensure reliable communications in this scenario we combine reception techniques based on distribution free statistical tests with sequential decoding on syndrome trellises. It will be shown that the proposed approach can ensure relatively high transmission rate with reasonable complexity.

Keywords: Nonparametric detection, syndrome trellis, sequential decoding, decoding complexity.

\section{Introduction}

Reception techniques using nonparametric or distribution free test can be con-sidered to be promising candidates for communication systems operating under severe interference. However decoding algorithms that were proposed for communication systems employing those techniques have relatively high computational complexity. This paper deals with the possibility of using sequential decoding on syndrome trellises to solve this problem.

\section{A DHA FH CDMA system: Transmission and reception}

Let us consider a multiple access system in which $K$ active users transmit information via a channel split into $Q$ identical nonoverlapping subchannels. In what follows it will be assumed that information to be transmitted is encoded into a codeword of a $q$-ary $(n, k, d)$ block code $(q<Q)$. Whenever a user is to transmit a $q$-ary symbol it places 1 in the position of the vector $\bar{x}_{g}$ corresponding to the symbol in question within the scope of the mapping in use (in what follows it will be assumed that all the positions of the vector are enumerated from 1 to $Q$, moreover for the sake of simplicity and without loss of generality we shall assume that the 1 st subchannel 
corresponds to 0 , the 2 nd subchannel corresponds to 1 and so on). Thus each $q$-ary symbol to be transmitted is mapped into a weight 1 binary vector (the construction under consideration is the Kautz-Singleton construction for binary superimposed codes [1]). Then a random permutation of the aforesaid vector is performed and the resulting vector $\bar{\chi}_{g}=\pi_{g}\left(\bar{x}_{g}\right)$ is then transmitted by employing $Q$-ary Frequency Shift Keying (FSK) (permutations are selected equiprobably from the set of all possible permutations and the choice is performed whenever a symbol is to be transmitted).

Within the scope of a certain codeword reception the receiver is to receive $n$ signals corresponding to the codeword in question. Note that the receiver is assumed to be synchronized with transmitters of all users. Therefore all the permutations done within the scope of transmission of the codeword in question are known to the user. The receiver measures energies at the outputs of all subchannels (let us designate the vector of the measurements corresponding to the $g$-th symbol as $\bar{\beta}_{g}$ ) and applies inverse permutation to each vector $\bar{\beta}_{g}$ corresponding to the respective symbol thus reconstructing the initial order of elements and obtaining vector $\bar{b}_{g}=\pi_{g}^{-1}\left(\bar{\beta}_{g}\right)$. Let us consider a matrix $B=\left[\bar{b}_{1}, \bar{b}_{2}, \ldots, \bar{b}_{n}\right]$ corresponding to the codeword of the inner code. Let us consider the submatrix $Y=\left[\overline{y_{1}}, \overline{y_{2}}, \ldots, \overline{y_{n}}\right]$ (here $Y$ is the submatrix corresponding to the $q$ first rows of the matrix $B$ and each vector $\overline{y_{g}}$ is the height $q$ column vector corresponding to the $g$-th symbol of the codeword). Please note that $Y$ provides all the information necessary to decode the codeword of the inner code.

\section{Nonparametric detection}

Let us now consider the detection problem. This problem can be decomposed into two successive stages: reliability values computation and decoding. First and foremost let us consider the first problem.

The aim of the first stage is to compute the decision reliability values for each symbol. The latter are to be used by the decoder at the second stage. Since the a priori presupposition is that each symbol can take any of the $q$ possible values for each symbol the corresponding vector of reliability values will be computed for each symbol.Thus a matrix of reliably values corresponds to each codeword. Since the matrix in question is used to make a decision on the transmitted codeword it will be further on referred to as decision statistics matrix $M^{D}$.

A number of reception techniques tolerant to severe jamming were developed in recent decades. In Normalized Envelope Detection (NED) method the decision statistics matrix is obtained by dividing each column of the matrix of envelopes by the sum of the respective column.

$$
M^{D}(i, j)=Y(i, j) / \sum_{j=1}^{q} Y(i, j) .
$$

However this method is hardly suitable for the case when the interfering users' signals have power much higher than that of the user under consideration. For this case a more robust method is needed. Hereinafter we shall consider some methods based on ordered statistics calculation. For simplicity let us assume that all elements of $Y$ are distinct, i.e., 


$$
\begin{aligned}
& \forall i \neq i^{\prime}, \forall j \neq j^{\prime}, \\
& Y(i, j) \neq Y\left(i^{\prime}, j\right), \\
& Y(i, j) \neq Y\left(i, j^{\prime}\right),
\end{aligned}
$$

and consider the indicator function

$$
\mathfrak{I}(x, y)=\left\{\begin{array}{l}
1 \text { if } x<y, \\
0 \text { if } x \geq y .
\end{array}\right.
$$

For each element of the matrix $Y$ its rank is given by

$$
R(t, z)=\sum_{t^{\prime} \neq t} \sum_{z \neq z^{\prime}} \Im\left(\left(Y_{M}(t, z), Y_{M}\left(t^{\prime}, z^{\prime}\right)\right)\right)+1 .
$$

In "rank sum method" [5] the rank matrix is the matrix of the decision statistics. Hereinafter we propose to combine the two previously considered methods by applying normalization to the rank matrix. In the resulting method that will be further on referred to as Normalized Rank Sum (NRS) the matrix of the normalized ranks is given by

$$
\tilde{R}(t, z)=R(t, z) / \sum_{z=1}^{q} R(t, z) .
$$

Within the scope of the proposed method the matrix of the normalized ranks is used as a decision matrix, i.e.,

$$
M^{D}(t, z)=\tilde{R}(t, z),
$$

and the decision matrix for the logarithmic version of the decoder is then given by

$$
\Lambda^{D}(t, z)=\log (\tilde{R}(t, z)) .
$$

As far as the second stage of the detection process (i.e., the decoding) is concerned the majority of papers that consider ordered statistics-based reception techniques (e.g., [5, 6]) use the decoding algorithms that boil down to exhaustive search. Unfortunately due to complexity considerations this approach leads to low rate codes. In [7] another approach has been proposed that is based on employing convolutional inner codes and Viterbi decoding. However the effectiveness of this approach is limited since the complexity of non-binary convolutional codes Viterbi decoding depends exponentially on the overall constraint length. Thus only codes with relatively small overall constraint length are practical. Both approaches can be explained in terms of the logarithmic version of the decision matrix, i.e., the decoder chooses codeword number

$$
i^{*}=\arg \max _{i \in C}\left(\sum_{t=1}^{n} \sum_{z=1}^{q}\left(\Lambda^{D} \cdot X_{i}\right)\right),
$$

to be the decoded codeword. Here $\cdot$ stands for Hadamard product, $C$ is the error correction code in use, $X_{i}$ is the matrix corresponding to the $i$-th codeword and $\Lambda^{D}$ is given by (7). Thus both methods make use of the decision matrix $M^{D}$ treating each (say $t$-th) column of the matrix as a vector of estimates of the conditional probabilities for the respective ( $t$-th) symbol of the codeword. However as has been mentioned above both approaches have relatively high complexity and thus are practical only for low rate code. In this paper another approach is proposed. In what follows we 
shall consider the perspective of decoding block codes on syndrome trellises by applying the sequential decoding concept.

\section{Using ZJ decoding algorithm for nonparametric detection: The proposed approach}

Syndrome trellises were introduced by B ahl et al. [2]. Unfortunately both APP decoding (that has been proposed in [2]) and Viterbi decoding on syndrome trellis [8] have complexity exponential with the number of parity check symbols. Thus only non-binary codes with relatively small distances can be decoded in this way. In what follows we propose another approach based on the classical sequential decoding paradigm. In particular we adapt the classical stack algorithm that has been proposed by Zigangirov [3] and Jelinek [4] for convolutional codes (also known as $\mathrm{ZJ}$ algorithm) for block codes decoding. To do so we use the syndrome trellis of the code in use and use the matrix $M^{D}$ (6) as the matrix of conditional probabilities for the respective symbols. The algorithm operates almost in the same way as the ZJ algorithm does and makes use of the conventional Fano metric. Let us consider a length $l$ path that corresponds to a vector $v^{i}(l)=\left[v_{0}{ }^{i}, v_{1}{ }^{i}, \ldots, v_{l-1}{ }^{i}\right]$ such that $v^{i}=\left[v_{0}{ }^{i}, v_{1}{ }^{i}, \ldots, v_{n-1}{ }^{i}\right]$ is a codeword of the code $C(n, k)$ in use (i.e., $\left.v^{i} \in C\right)$. The metric for the respective path will then be given by

$$
\mu\left(v^{i}(l)\right)=\sum_{t=0}^{l-1}\left(\log \left(\frac{\sum_{j=0}^{q-1}\left(M^{D}(t+1, j+1) X_{i}(t+1, j+1)\right)}{\sum_{j=0}^{q-1}\left(p(j+1) M^{D}(t+1, j+1)\right)}\right)-\Re\right),
$$

where $p(j+1)$ is the probability of the fact that the respective symbol has been transmitted and $\mathfrak{R}$ is the rate of the code in use. The algorithm terminates when the winning path has full length. Then all the paths having full length $n$ are extracted from the stack and exhaustive search is applied to the resulting list (in what follows we shall call it the final list). Again the matrix $M^{D}$ is treated as the conditional probabilities matrix, i.e., if $I$ is the set of numbers of the $(n, k)$ block code codewords that were included in the resulting list and $X_{i}(i \in I)$ are the respective Kautz-Singleton matrices then the decoder declares the codeword with the number

$$
i^{*}=\arg \max _{i \in I}\left(\sum_{t=1}^{n} \sum_{z=1}^{q}\left(\Lambda^{D} \cdot X_{i}\right),\right.
$$

to be the decoded codeword.

\section{Simulation scenario}

To investigate the effectiveness of the proposed algorithm the following scenario will be considered: It will be assumed that the user under consideration transmits in a system with $Q$ orthogonal subcarriers employing the transmission technique 
considered above and apart from the user under consideration $K$ interfering signals are transmitted in the system under consideration. Hereinafter it will be assumed that each interfering signal has the same form as that of the user under consideration but its power at the receiver end is $\kappa$ times higher than that of the signal of the user under consideration. Moreover it will be assumed that apart from narrowband interfering signals the received signal is influenced by the wideband interference that will be modeled as an Additive White Gaussian Noise characterized by Signal-to-Noise Ratio $\mathrm{SNR}=10 * \log _{10}\left(\frac{E_{s}}{E_{N}}\right)$ where $E_{s}$ is the energy of the signal transmitted by the user under consideration (at the receiver side), and $E_{N}$ is noise energy (please note that $E_{N}$ is noise energy in the entire band whereas $E_{s}$ is the energy in the effective band occupied by the transmitted signal. Since the effective bandwidth is much smaller than the entire one SNR can take great negative values). In particular hereinafter we shall consider the case when $q=8, Q=4096, \kappa=10^{4}$, SNR $=-20 \mathrm{~dB}$ and systematic MDS code $C(6,3)$ over $\operatorname{GF}(8)$. In what follows we shall investigate the performance of the proposed detector for different numbers of interfering users $K$.

\section{Simulation: Results}

In Fig. 1 Frame Error Rate (i.e., the probability of erroneous decoding per block) ensured by the proposed decoder (Sequential Decoder (SD)) for different values of $K$ (number of interfering users) is shown in Fig. 1 (curve for the Exhaustive Search decoder (ES) employing the same decision statistics matrix is shown in the same figure).

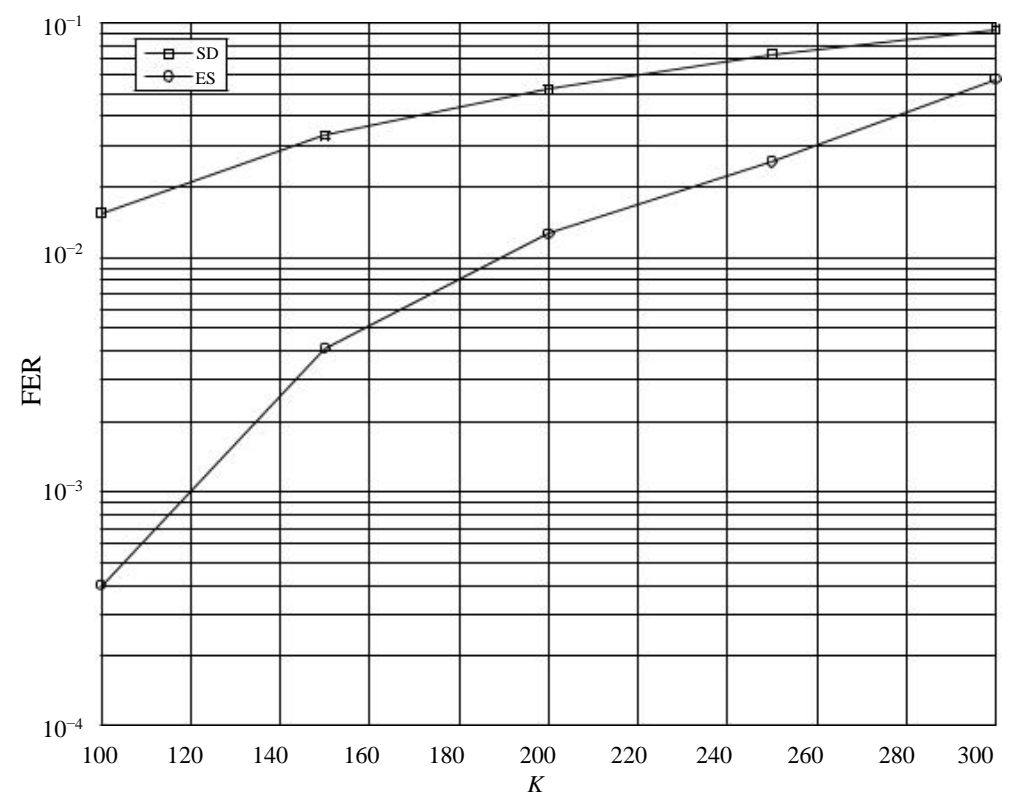

Fig. 1. FER for the proposed decoder (SD) and the exhaustive search decoder (ES)) 
As can be seen from the presented curves even though the proposed algorithm shows considerable performance degradation as compared to the exhaustive search algorithm the probability of error per block is still low enough to use the proposed decoder as inner decoder in a cascaded coding scheme. It should be noted that the complexity of the exhaustive search even for our example is prohibitively large. Thus unlike the proposed solution its counterpart that uses exhaustive search can hardly be used in practical systems.

One of the most serious issues that is commonly associated with practical implementation of the sequential decoding is the problem of the stack size. In classical ZJ algorithm the stack has been assume to be infinite. In real life scenarios this is infeasible. Thus it is interesting to investigate the performance of the decoding algorithm that uses stack of a fixed size. In Fig. 2 Frame Error Rate (i.e., the probability of erroneous decoding per block) ensured by the proposed decoder (Sequential Decoder (SD)) for different values of $K$ (number of interfering users) and different values of the Stack Size (StS) is shown.

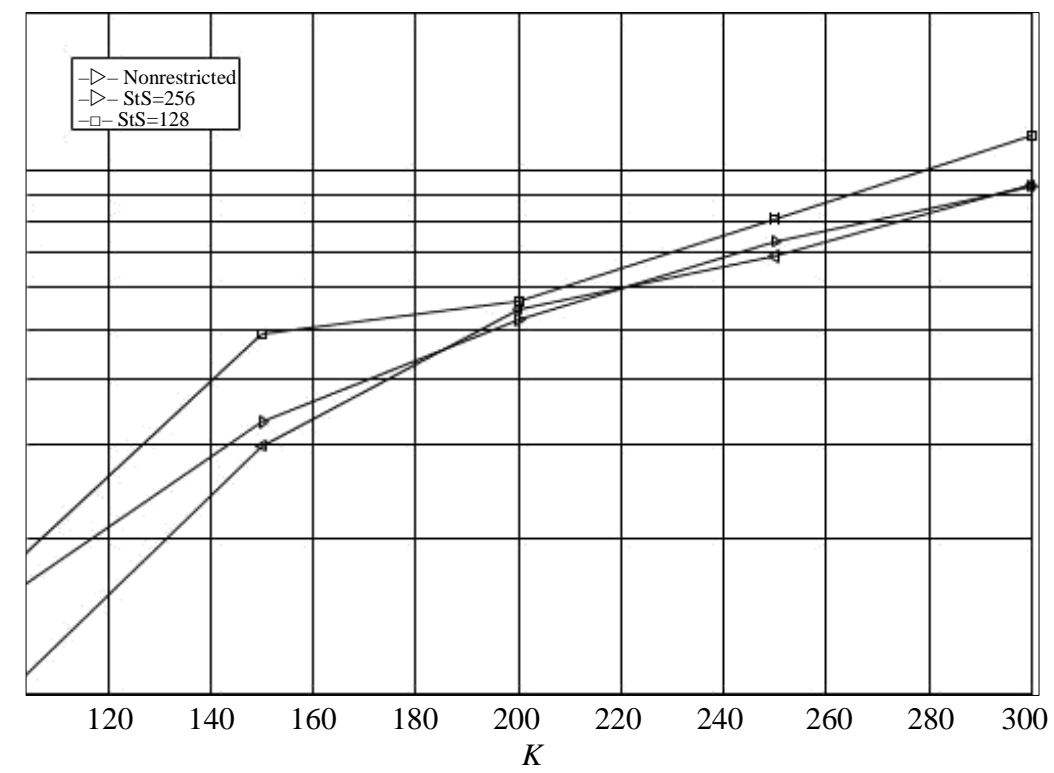

Fig. 2. FER for the proposed decoder (SD) and different sizes of the stack

Let us note that performance of the decoder using a fixed size stack is very close to that of the decoder that uses infinite stack even if the size of the stack is moderate.

As has been pointed out in Section4 the main reason for using the sequential decoder is the complexity of the decoding. In general the complexity of the decoding depends on the number of nodes visited by the decoding algorithm and thus it is a random value. In tables 1 and 2 the maximal and average number of nodes visited by the decoder are given for for different values of $K$ (number of interfering users) and different values of the Stack Size (StS) are given.

It can be noted that the stack size restriction has almost no effect on the number of nodes visited by the decoder. The maximal (worst case) number of nodes is about 
1.5 times less that the maximum number of nodes in a syndrome trellis layer (the latter is given by $N_{\max }=q^{k}$ ) while the average number of nodes that are to be visited is still 2 times less. Thus the proposed decoder indeed offers certain complexity reduction. Another aspect that affects the complexity is the size of the final list. The maximum values of the final stack size for different values of $K$ (number of interfering users) and different values of the StS are presented in Table 3.

Table 1. Number of nodes visited (maximal) for different sizes of the stack

\begin{tabular}{|c|c|c|c|c|c|}
\hline StS & \multicolumn{5}{|c|}{ Number of interfering users } \\
\hline Stack size & 300 & 250 & 200 & 150 & 100 \\
\hline Not restricted & 345 & 349 & 364 & 363 & 380 \\
\hline 256 & 327 & 381 & 331 & 415 & 385 \\
\hline 128 & 353 & 369 & 347 & 359 & 415 \\
\hline
\end{tabular}

Table 2. Number of nodes visited (average) for different sizes of the stack

\begin{tabular}{|c|c|c|c|c|c|}
\hline StS & \multicolumn{5}{|c|}{ Number of interfering users } \\
\hline Stack size & 300 & 250 & 200 & 150 & 100 \\
\hline Not restricted & 119.9056 & 123.7721 & 124.4617 & 125.6317 & 118.0990 \\
\hline 256 & 119.8394 & 121.3169 & 124.9358 & 126.6210 & 118.8940 \\
\hline 128 & 122.1334 & 120.3910 & 124.7390 & 126.1415 & 118.6147 \\
\hline
\end{tabular}

Table 3. Size of the final list

\begin{tabular}{|c|l|l|l|l|l|}
\hline StS & \multicolumn{5}{|c|}{ Number of interfering users } \\
\hline Stack size & 300 & 250 & 200 & 150 & 100 \\
\hline Not restricted & 39 & 36 & 39 & 49 & 53 \\
\hline 256 & 39 & 47 & 42 & 53 & 55 \\
\hline 128 & 36 & 35 & 34 & 40 & 41 \\
\hline
\end{tabular}

One can notice that even the worst case final list size is moderate. Moreover there is almost no dependency between the final list size and the size of the stack.

\section{Modified reception strategy}

One of the reasons for the fact that the resulting Frame Error Rates for the proposed algorithm and the exhaustive search decoding algorithm are relatively high is the influence of the interfering narrowband signals. Although the rank calculation makes the decision statistics less sensitive to the distortion introduced by these signals they still have some impact. Therefore we suggest a modified version of the decision statistics matrix that is calculated by clipping the rank matrix prior to normalization. This method will be further on referred to as Normalized Clipped Rank Sum. The clipped rank matrix is given by

$$
C_{R}(t, z)=\left\{\begin{array}{l}
\theta(R), \quad R(t, z) \geq \theta(R), \\
R(t, z), R(t, z)<\theta(R),
\end{array}\right.
$$

where $\theta(R)$ is the clipping threshold (the designation means that the threshold can depend on the matrix $R$ ). The normalized clipped rank matrix is given by 


$$
\tilde{C}_{R}(t, z)=C_{R}(t, z) / \sum_{z=1}^{q} C_{R}(t, z) .
$$

The decision matrix is then given by

$$
M_{C}^{D}=\tilde{C}_{R}(t, z),
$$

and the decision matrix for the logarithmic version of the decoder is then given by

$$
\Lambda_{C}^{D}(t, z)=\log \left(C_{R}(t, z) / \sum_{z=1}^{q} C_{R}(t, z)\right),
$$

in what follows we use the following way to define the threshold. Let $\theta_{i}$ be the maximum value for the $i$-th column of the matrix $R(t, z)$,

$$
\theta_{i}=\max _{z=\{1, \ldots, q\}}(R(i, z)),
$$

the threshold value $(R)$ is then given by

$$
\theta(R)=\min _{i=\{1, \ldots, n\}} \theta_{i} .
$$

\section{Simulation: Results for the modified version}

Let us now consider the simulation results. We consider the same simulation scenario that has been described in Section 5. The Frame Error Rate for the proposed decoder (SD) and the exhaustive search (ES) decoder for different values of $K$ is shown in Fig. 3. In Fig. 4 Frame Error Rate (i.e., the probability of erroneous decoding per block) ensured by the proposed decoder (sequential decoder (SD)) for different values of $K$ (number of interfering users) and different values of the stack size (StS) is shown. Finally in Tables 4, 5, and 6 the maximal and average number of nodes visited by the decoder and the final list size are given for different values of $K$ (number of interfering users) and different values of the stack size (StS) for the modified reception strategy are presented.

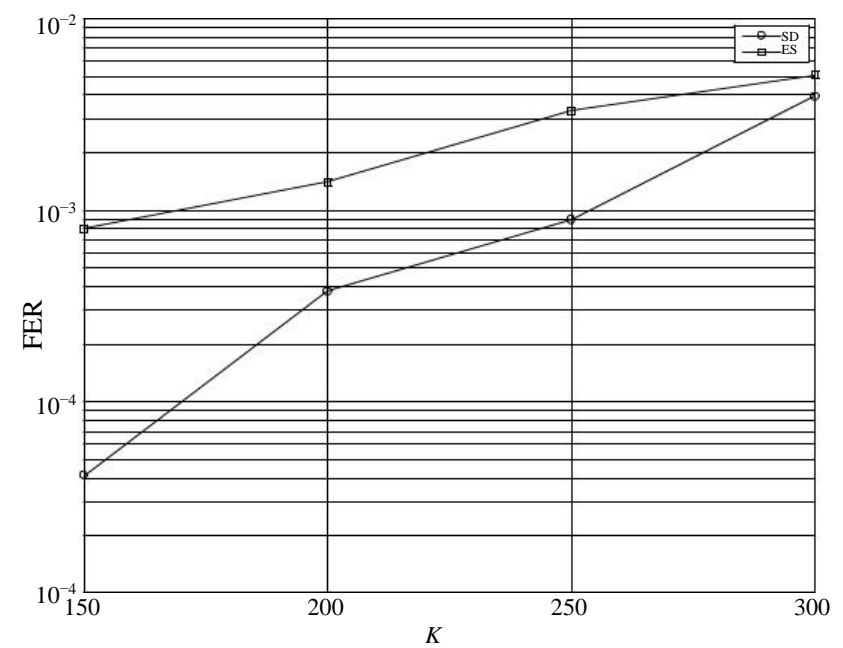

Fig. 3. FER for the proposed decoder (SD) and the exhaustive search decoder (ES)) using the modified strategy 


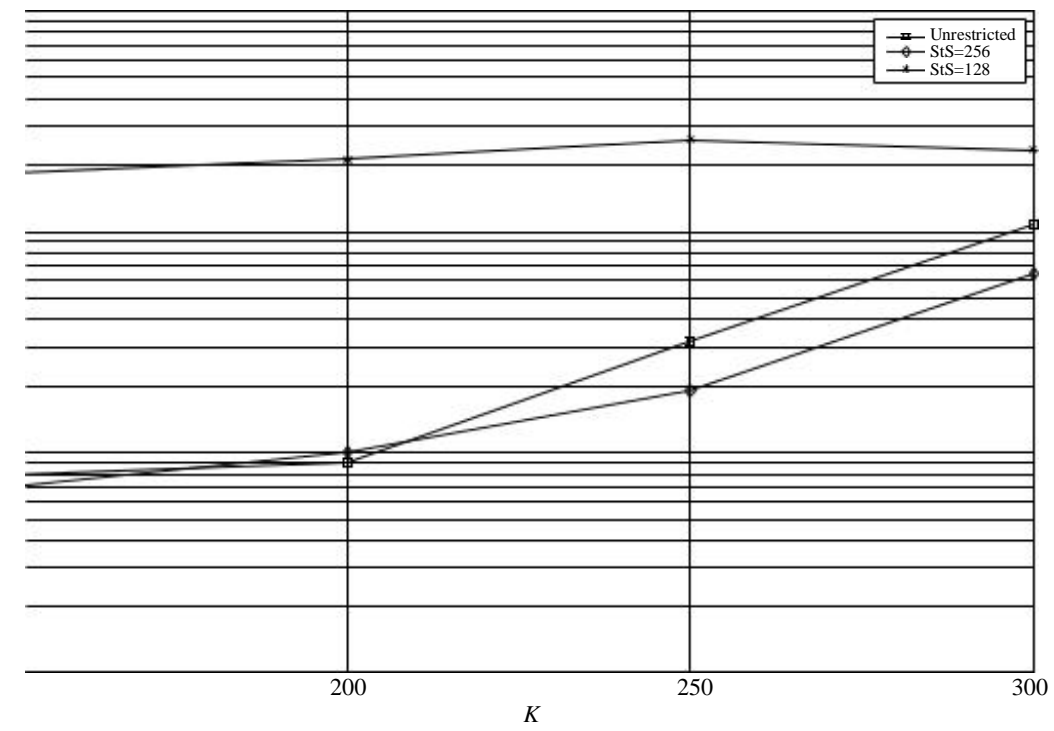

Fig. 4. FER for the proposed decoder (SD) and different sizes of the stack for the modified strategy

Table 4. Number of nodes visited (maximal) for different sizes of the stack (for the modified reception strategy)

\begin{tabular}{|c|c|c|c|c|}
\hline StS & \multicolumn{4}{|c|}{ Number of interfering users } \\
\hline Stack size & 300 & 250 & 200 & 150 \\
\hline Not restricted & 622 & 683 & 690 & 644 \\
\hline 256 & 597 & 657 & 667 & 716 \\
\hline 128 & 472 & 412 & 429 & 490 \\
\hline
\end{tabular}

Table 5. Number of nodes visited (average) for different sizes of the stack (for the modified reception strategy) different values of the stack size (StS) for the modified reception strategy are presented.

\begin{tabular}{|c|c|c|c|c|}
\hline StS & \multicolumn{4}{|c|}{ Number of interfering users } \\
\hline Stack size & 300 & 250 & 200 & 150 \\
\hline Not restricted & 189.9046 & 189.1874 & 192.5638 & 194.9336 \\
\hline 256 & 187.6872 & 190.3603 & 193.5807 & 195.3684 \\
\hline 128 & 184.7402 & 188.0763 & 193.1795 & 189.8682 \\
\hline
\end{tabular}

Table 6. Size of the final list

\begin{tabular}{|c|c|c|c|c|}
\hline StS & \multicolumn{4}{|c|}{ Number of interfering users } \\
\hline Stack size & 300 & 250 & 200 & 150 \\
\hline Not restricted & 103 & 119 & 128 & 112 \\
\hline 256 & 95 & 122 & 120 & 165 \\
\hline 128 & 75 & 67 & 87 & 88 \\
\hline
\end{tabular}

Comparing Fig. 3 with Fig. 1 one can notice that the rank matrix clipping actually results in a substantial performance gain. However this gain comes at the expense of more than twofold growth of the maximal (worst case) growth of the number of nodes visited. The average number of the nodes visited grows as well (even though the increase in the average number of nodes is much less substantial). The size of the 
final list also is 2 to 3 times larger. Thus we can conclude that the modified strategy usage results in complexity increase. Moreover as can be seen from Fig. 3 and Fig. 4 the usage of the proposed modification results in error floor performance for moderate numbers of interfering users and makes the decoder more sensitive to the stack size restriction (moderate stack size results in sufficient performance decrease). Thus it is advisable to use the modified reception strategy for the low rate codes decoding that can be performed by exhaustive search.

\section{Conclusion}

Hereinabove the problem of decoding nonbinary block codes by using a sequential decoding on a syndrome trellis has been considered. Two decoding reception strategies (the Normalized Rank Sum and Normalized Clipped Rank Sum) and a decoding algorithm that is based on the classical Zigangirov-Jelinek algorithm were proposed. It has been shown that Normalized Rank Sum method can be used in combination with the proposed algorithm to decode relatively high rate codes in the cascaded coding construction, whereas the Normalized Clipped Rank Sum is better suited for the decoding of lower rate codes by exhaustive search.

Acknowledgements: The results in Sections 7 and 8 were obtained by Dmitry Osipov at the IITP RAS and financed by the Russian Science Foundation Grant (Project No 14-50-00150).

\section{References}

1. K a u t z, W. H., R. C. S i n g l e t o n. Nonrandom Binary Superimposed Codes. - IEEE Transactions on Information Theory, Vol. 10, 1964, No 4, pp. 363-377.

2. B a h 1, L. R., J. C o c k e, F. J e 1 i n e k, J. R a v i v. Optimal Decoding of Linear Codes for Minimizing Symbol Error Rate. - IEEE Transactions on Information Theory, Vol. 20, 1974, No 2, pp. 284-287.

3. Z i g a $\mathrm{n}$ g i r o v, K. S. Some Sequential Decoding Procedures. - Problems of Information Transmission, Vol. 4, 1966, No 2, pp. 1-10.

4. J e 1 i n e k, F. A Fast Sequential Decoding Algorithm Using a Stack. - IBM Journal of Research and Development, Vol. 13, 1969, No 6, pp. 675-685.

5. V i s w a n a t h a n, R., S. C. G u p t a. Nonparametric Receiver for FH-MFSK Mobile Radio. IEEE Transactions on Communications, Vol. 33, 1985, No 2, pp. 178-184.

6. K o n d r a s h o v, K., V. A f a n a s s i e v. Ordered Statistics Decoding for Semi-Orthogonal Linear Block Codes over Random Non-Gaussian Channels. - In: Proc. of 13th International Workshop on Algebraic and Combinatorial Coding Theory, Pomorie, Bulgaria, 15-21 June 2012, pp. 192-196.

7. O s i p o v, D. Inner Convolutional Codes and Ordered Statistics Decoding in a Multiple Access System Enabling Wireless Coexistence. - In: Proc. of 6th International Workshop, MACOM'2013, Vilnius, Lithuania, 16-17 December 2013, pp. 33-38.

8. W o 1 f, J. Efficient Maximum Likelihood Decoding of Linear Block Codes Using a Trellis. - IEEE Transactions on Information Theory, Vol. 24, 1964, No 1, pp. 76-80. 